Revista de Investigación Educativa 26

enero-junio, 2018 | ISSN 1870-5308 | Xalapa, Veracruz

Instituto de Investigaciones en Educación | Universidad Veracruzana

\title{
Enseñanza y difusión sobre el uso de recursos educativos abiertos con MOOC: un estudio de caso
}

\section{Teaching and dissemination about the use of open educational resources with MOOC: a case study}

\author{
Martín Alonso Mercado Varela ${ }^{a}$ \\ Katiuska Fernández Morales ${ }^{b}$ \\ Gilles Lavignec \\ María Soledad Ramírez Montoya ${ }^{d}$
}

Recibido: 24 de agosto de 2016 Aceptado: 07 de febrero de 2017

El presente artículo analiza el caso de un Curso en Línea Masivo y Abierto (MOоc) que tiene por objetivo desarrollar competencias digitales y de diseño instruccional para integrar Recursos Educativos Abiertos (REA) en ambientes de aprendizaje. Se utiliza la metodología del estudio de caso para realizar los procesos de investigación. El artículo tiene como objetivo analizar la difusión y los resultados de aprendizaje obtenidos de la implementación del Mоос, para lo cual se analizan distintas fuentes de información, tales como registros de navegación, respuestas a encuestas y evidencias de aprendizaje. Se concluye que el MOOC analizado funciona como un espacio efectivo para la enseñanza y difusión sobre los REA.

Palabras clave: Desarrollo de competencias; recursos educacionales; e-learning; educación a distancia.

\footnotetext{
a Doctor en Ciencias Educativas, Posdoctorante, Escuela de Humanidades y Educación, Tecnológico de Monterrey, México.

b Doctora en Investigación Educativa, Investigadora, Instituto de Investigación y Desarrollo Educativo, Universidad Autónoma de Baja California, México.

c Doctor en Filosofía, Investigador, Instituto de Investigación y Desarrollo Educativo, Universidad Autónoma de Baja California, México.

d Doctora en Filosofía y Ciencias de la Educación, Profesora-Investigadora, Escuela de Humanidades y Educación, Tecnológico de Monterrey, México.
} 
This paper analyzes the case of a Massive Open Online Course (MOоc) which aims to develop digital competencies and instructional design to integrate Open Educational Resources (OER) into learning environments. The methodology of the case study is used to carry out the research processes. The article aims to analyze the diffusion and learning outcomes of the implementation of the MOOc, which discussed various sources of information, such as navigation logs, responses to surveys and evidence of learning. It is concluded that the analyzed MOOc, functioned as an effective space for teaching and diffusion about OER.

Keywords: Skills development; educational resources; e-learning; distance education.

\title{
Enseñanza y difusión sobre el uso de recursos educativos abiertos con Mooc: un estudio de caso
}

\author{
Teaching and dissemination about the use of open \\ educational resources with MOoc: a case study
}

\section{Introducción}

1 1 Movimiento Educativo Abierto se define como aquellas actividades educativas Recursos Educativos Abiertos (REA), así como la diseminación de prácticas educativas en entornos académicos, gubernamentales, institucionales, entre otros (Ramírez, 2013). Este movimiento está integrado por distintas iniciativas, tales como el uso de repositorios abiertos (Yiotis, 2005), el código abierto (Lakhan \& Jhunjhunwala, 2008) y el OpenCourseWare del Massachusetts Institute of Technology (MIT), el cual creó un catálogo de materiales educativos en formato abierto para todo el público interesado (Atkins, Brown \& Hammond, 2007).

Con el advenimiento de los REA auspiciados por la Organización de las Naciones Unidas para la Educación, la Ciencia y la Cultura (UnesCo), el movimiento ha con- 
tado con el apoyo de políticas diseñadas desde instituciones internacionales que facilitan la democratización del conocimiento, lo cual representa su principal objetivo. Con el antecedente del OpenCourseWare del MIT, iniciativa que tuvo su aparición en 2001, un mayor número de instituciones educativas ofreció en forma gratuita y abierta materiales pedagógicos. Con esto en mente, la UNESCO organizó en 2002 el primer foro mundial sobre recursos educativos de libre acceso, donde se adoptó el término REA (UNESCO, 2002).

Un REA puede ser definido como "la disposición abierta de recursos educativos, habilitados por tecnologías de la información y la comunicación, para la consulta, uso y adaptación por una comunidad de usuarios con fines no comerciales" (UNESCO, 2002, p. 24). De igual modo, es concebido como recursos de enseñanza, aprendizaje e investigación que son del dominio público o han sido puestos en libertad, en virtud de una licencia de propiedad intelectual que permite su uso libre o re-propositivo por otros, que incluye recursos tales como cursos completos, materiales de curso, libros, videos, exámenes, software y cualquier otra herramienta, material o técnicas usadas para apoyar el acceso al conocimiento (Atkins et al., 2007).

Pese a la importancia que ha adquirido el movimiento, su adopción no ha sido global. Aun cuando los REA tienen el potencial de abrir el acceso a recursos educativos y a educación de calidad, mientras que reducen costos para el aprendizaje formal e informal, muchas instituciones educativas siguen sin hacer uso de estas potencialidades. Aunque el impacto del Movimiento Educativo Abierto es global, las iniciativas y universidades que se han sumado y fomentan este tipo de prácticas provienen principalmente de Estados Unidos y Europa. En este sentido, la participación de Latinoamérica, aunque ha presentado grandes avances, queda limitada en comparación. Es necesario llevar al movimiento de la etapa de compartir el conocimiento a través de las Tecnologías de la Información y la Comunicación (TIC), hacia una más compleja, representada por las Prácticas Educativas Abiertas (PEA) para mejorar procesos formativos, de gestión e investigación (Ramírez, 2015). Ehlers (2011) define a las PEA como:

Aquellas prácticas que soportan el uso, reutilización y producción de Recursos Educativos Abiertos de alta calidad, a través de políticas institucionales que promuevan modelos pedagógicos innovadores y empoderen a los aprendices como co-productores de su propia ruta de aprendizaje a lo largo de la vida. (p.3)

Aun con el diseño de políticas e iniciativas promovidas para impulsar el uso de los REA, permanece una serie de retos que dificultan su adopción. Retos asociados con modelos de sostenibilidad, preservación del acceso, derechos de autor, contenidos de 
calidad y sobre la estructura tecnológica para poner en práctica las iniciativas (Atkins et al., 2007; McAndrew \& Farrow, 2013), son parte del trabajo que queda por hacer para impulsar el uso de REA. Por otro lado, Ehlers (2011), Deimann y Farrow (2013) y McAndrew (2011), sostienen que el movimiento se ha centrado en el contenido (REA) y no en las PEA, necesarias para utilizar el contenido. De tal manera, resulta importante no sólo difundir el conocimiento sobre los REA, sino también la enseñanza sobre cómo utilizar este tipo de recursos en la práctica educativa.

Ante la gama de competencias y habilidades que se pueden nombrar en el Movimiento Educativo Abierto, se identifican como principales: la producción, búsqueda y selección, difusión y movilización (uso del recurso) de REA, especialmente en el contexto en que son utilizadas por profesores y aprendices. Al analizar los alcances que puede tener un REA, estas competencias son fundamentales para lograrlos. De acuerdo con Wiley (2010), el adjetivo abierto de un REA implica una licencia de autor que permite reutilizar (el contenido de forma inalterada), revisar (adaptar, modificar o alterar el contenido), mezclar (combinar el contenido original creando algo nuevo) y redistribuir (compartir el contenido con otras personas).

Dentro de las iniciativas del Movimiento Educativo Abierto, los Cursos en Línea Masivos y abiertos (MOOC), representan un espacio para la enseñanza. Los MOOC son considerados una nueva opción en el repertorio de la educación en línea (Guárdia, Maina \& Sangrá, 2013; Liyanagunawardena, Adams \& Williams, 2013). Estos cursos integran distintas características del Movimiento Educativo Abierto, tales como gratuidad y accesibilidad, posibilitando el ingreso de participantes y el uso y compartición de REA; aunque la compartición del trabajo intelectual que se crea o pone a disposición dentro de un espacio MOoc, no es una práctica generalizada. En consecuencia, los MOOC se constituyen como un espacio en potencia para la enseñanza y movilización de REA.

De tal manera, el presente artículo tiene por objetivo analizar la enseñanza y difusión del Movimiento Educativo Abierto, en particular, sobre los REA, para lo cual se analizó el caso de un Mooc implementado en la plataforma Coursera.

\section{Caso de análisis}

El curso Innovación educativa con recursos abiertos representa el caso de análisis. En las líneas siguientes se describen las características principales asociadas al curso. 
Objetivo general del curso: el participante identificará estrategias en el uso de REA a través del análisis de su contexto, las aportaciones de la temática en el ámbito teórico y las experiencias que se han suscitado en el ámbito académico, con el fin de contribuir con prácticas innovadoras en el movimiento educativo abierto.

Organización del curso: tiene una duración de 4 semanas en las que se solicita al participante una actividad de entre 4-6 horas semanales donde se desarrollan ciertas temáticas y competencias (Tabla 1). En la dinámica semanal de actividades se establecen cuatro actividades principales, que consisten en 1) participar en encuestas de opinión, 2) analizar videos, lecturas y materiales, 3) evidenciar el aprendizaje en portafolios y socializarlo en los espacios del curso y 4) autoevaluar el aprendizaje.

\section{Tabla 1. Temáticas y competencias asociadas}

\begin{tabular}{cll}
\hline Semana & \multicolumn{1}{c}{ Temática } & \multicolumn{1}{c}{ Competencia } \\
\hline 1 & $\begin{array}{l}\text { Movimiento educativo } \\
\text { abierto }\end{array}$ & $\begin{array}{l}\text { Identificar en el ambiente de trabajo posibles innovacio- } \\
\text { nes educativas por medio de la integración de REA }\end{array}$ \\
\hline 2 & $\begin{array}{l}\text { Búsqueda y uso de recursos } \\
\text { educativos abiertos }\end{array}$ & $\begin{array}{l}\text { Buscar, seleccionar y evaluar REA para su integración en } \\
\text { ambientes educativos enriquecidos con el uso de TIC }\end{array}$ \\
\hline 3 & $\begin{array}{l}\text { Visibilidad y diseminación } \\
\text { abierta del conocimiento }\end{array}$ & $\begin{array}{l}\text { Diseñar estrategias de difusión de la producción científica } \\
\text { y académica para potenciar la transferencia de la } \\
\text { innovación educativa con REA }\end{array}$ \\
\hline 4 & $\begin{array}{l}\text { Movilización de prácticas } \\
\text { educativas abiertas en } \\
\text { ambientes de aprendizaje }\end{array}$ & $\begin{array}{l}\text { Diseñar actividades para utilizar REA considerando la } \\
\text { producción, utilización y reutilización en ambientes } \\
\text { académicos e institucionales }\end{array}$ \\
\hline
\end{tabular}

Fuente: Elaboración propia.

La comunicación e interacción se realizó a través de un proceso de socialización con ayuda de las siguientes estrategias:

- Facilitadores: desempeñan el papel de propiciar las conexiones de aprendizaje entre participantes. El equipo se compone de dos facilitadores docentes (responsables del curso), dos de apoyo (elegidos por los responsables del curso) y de facilitadores voluntarios (fueron elegidos de acuerdo con su experiencia en procesos de facilitación remota y conocimiento sobre la temática del curso). Los voluntarios tienen una doble función, ya que también son estudiantes del curso. 
- Foros de discusión académicos y administrativos: los de tipo académico son espacios dedicados a la construcción conjunta de conocimientos, se espera que en un ambiente de respeto y armonía se colabore compartiendo puntos de vista y opinión. Los administrativos son espacios dedicados a resolver dudas, problemas e inquietudes del curso de índole logística, tecnológica y/o administrativa.

- Participación en sesiones en vivo: dos sesiones en vivo con los facilitadores docentes del curso. Las sesiones se transmitieron por el canal de Youtube del curso (https://www.youtube.com/channel/UCr5tK3lgu8W7uDr23ktexmg/videos) a través de la modalidad de Google Hangout en Vivo. Tienen el objetivo de discutir las principales inquietudes surgidas en cada semana, así como orientar la actividad de los participantes.

- Grupos de autoestudio: espacio virtual de autogestión por los propios miembros (participantes del curso), de carácter informal y sin moderación de facilitadores. Los miembros tienen un objetivo en común, ayudarse mutuamente en la resolución de dudas e inquietudes y en la profundización de conocimiento. Este espacio busca ofrecer la posibilidad de organización de grupos de autoestudio locales (y/o presenciales), en donde los participantes interactúen entre sí de forma independiente y apoyándose colaborativamente.

- Redes sociales: se incentiva el uso de redes sociales con distintos objetivos asociados a las actividades que enmarca el curso. Twitter.com: socializar y compartir sus ideas, crear tendencias; Bitly.com, Tiny.cc y Goo.gl: reducir hipervínculos o links de Internet; Slideshare.net y Scribd.com: compartir documentos y presentaciones.

Los recursos didácticos que se utilizaron para facilitar los procesos de enseñanzaaprendizaje se describen a continuación:

- Recursos educativos abiertos en distintos formatos: lecturas (libros y artículos), objetos de aprendizaje y videos; se consideran materiales de apoyo.

- Videos de expertos: en cada una de las semanas se conocerá la opinión de expertos a través de videos, con el fin de que el participante esté al tanto de las tendencias de académicos internacionales reconocidos en la temática. Los expertos fueron invitados para grabar los videos ex profeso para el curso.

La evaluación se realizó con las siguientes estrategias:

- Portafolios electrónicos: en cada una de las semanas el participante realiza una actividad específica donde coloca una evidencia digital de su aprendizaje, 
la cual debe socializar en redes sociales para incentivar la compartición de conocimiento y el aprendizaje colectivo. La evaluación se realiza por el método de pares. Cuatro tipos de portafolios son evaluados, a continuación se describe su función. El portafolio diagnóstico tiene por finalidad que el participante analice los contenidos del curso y los integre a sus conocimientos previos para hacer un autodiagnóstico inicial y donde visualice una innovación educativa que pueda realizar con REA; el objetivo del portafolio de trabajo es que el participante explore posibles estrategias de uso de REA y sobre el diseño de búsqueda de información y REA; con el portafolio de evaluación el participante debe valorar cómo está cubriendo los criterios de las estrategias de difusión de la producción científica y académica, en su capacidad para potenciar la transferencia de la innovación educativa con REA; y el portafolio de presentación tiene por meta que el participante exponga una evidencia donde considere explorar posibilidades de movilización, definiendo un plan de acción para movilizar REA en su contexto profesional de trabajo. El portafolio de presentación es la máxima evidencia de aprendizaje, representa un plan para utilizar REA en un ámbito de aprendizaje.

- Autoevaluación del aprendizaje: al finalizar cada una de las semanas, el participante valorará sus conocimientos por medio de autoevaluaciones y encuestas de opinión.

\section{Consideraciones metodológicas}

El presente trabajo se llevó a cabo bajo la metodología del estudio de caso. De acuerdo con Stake (1999), éste se define como el estudio de la particularidad y complejidad para comprenderlo en determinadas circunstancias. Se analizaron dos categorías del caso: 1) Difusión y 2) Aprendizaje. Al interior de la categoría difusión, se analizan indicadores tales como el número de participantes registrados en el curso, número de participantes activos (los que realizan las actividades del curso), niveles de participación y el origen de los participantes. Tiene por objetivo establecer los alcances que tuvo el curso en la difusión del conocimiento de los REA. Al interior de la categoría aprendizaje, se analizan indicadores tales como las expectativas, la satisfacción y la evaluación del aprendizaje. Tiene por objetivo informar sobre los resultados de aprendizaje que se lograron dentro del curso. 
La actividad de los investigadores se dividió en tres etapas generales: 1) Exploratoria: consistió en participar como facilitadores de aprendizaje para conocer a profundidad las características de la situación educativa, principalmente, la dinámica generada a partir de la propuesta de aprendizaje del curso. 2) Trabajo de campo: estuvo centrada en la aplicación de una encuesta al final de cada semana, que permitió conocer las expectativas y satisfacción de aprendizajes del participante. 3) Análisis: se realizó al término del curso, se analizaron las respuestas que los participantes proporcionaron a partir de las encuestas, se analizaron registros de navegación (estadísticos de participación en el curso) y se revisaron las evaluaciones de aprendizaje.

\subsection{Fuentes de información}

Los datos analizados para poder informar sobre las categorías de análisis del curso se obtuvieron a partir de distintas fuentes información. En las siguientes viñetas se describen dichas fuentes:

- Registros de navegación producidos por las interacciones de los participantes con la plataforma Coursera. El tablero de gestión de datos de la plataforma registra las distintas actividades que realizan los participantes y los espacios de socialización en los que interactúan. La manipulación del tablero de gestión permite obtener las estadísticas asociadas a la actividad de los participantes.

- Direcciones IP para establecer los lugares de conexión del participante (se obtienen por el tablero de gestión).

- Respuestas de los participantes a encuestas aplicadas durante el curso. Respondieron a cuatro encuestas durante el curso (una por semana), en las cuales se indagaba sobre los diferentes aprendizajes obtenidos por el participante al finalizar cada semana.

- Evaluaciones de las evidencias de aprendizaje (portafolios). Las evaluaciones se hicieron sobre los cuatro tipos de portafolios que se solicitaban al participante: diagnóstico, trabajo, evaluación y presentación.

\subsection{Análisis de datos}

Los datos que informan sobre la categoría de difusión son de naturaleza cuantitativa. Por medio del tablero de gestión de datos de la plataforma Coursera, se obtuvieron los estadísticos sobre esta categoría. El análisis y la presentación de los datos son de 
carácter descriptivo. Los datos corresponden al número de interacciones registradas del participante con las distintas actividades del curso.

Los datos que informan sobre la categoría aprendizaje son de naturaleza mixta. El corpus de las encuestas se analizó mediante un proceso cualitativo. Se hizo un análisis de contenido deductivo en tres ciclos de codificación. Para identificar las principales respuestas de los participantes, se utilizaron los códigos de a) producción, b) búsqueda y selección, c) difusión y d) movilización, que hacen referencia a las principales competencias asociadas a los REA, y el código e) otras competencias, que hace referencia a otro tipo de competencias en el uso de la tecnología y en los espacios de aprendizaje en línea. Fueron analizados 1200 comentarios en total.

Además, la evaluación de los portafolios se analizó mediante un proceso cuantitativo. La evaluación se realizó por el método de pares, por el cual cada portafolio fue evaluado por tres participantes asignados aleatoriamente por la plataforma del curso, así como por expertos en el tema (en parte por los investigadores). Los portafolios se evaluaron conforme a una rúbrica con tres niveles (insuficiente, aceptable y sobresaliente), que indican el fortalecimiento de un determinado conocimiento o habilidad. Fueron analizados 2,083 portafolios en total.

\section{Resultados}

\subsection{Difusión}

Se registraron en el curso 14,227 participantes provenientes de 101 países. México (39\%), España (14\%) y Colombia (10\%), contribuyeron en mayor medida en el número de participantes registrados (Figura 1). A nivel continente, América Latina registró aproximadamente $81 \%$, Europa, $18 \%$, Asia, $1 \%$, África, $0.3 \%$ y Oceanía, o.1\%. Para matizar la difusión del curso, se tomó en consideración la participación activa. Es así que, del total de participantes, únicamente 6,716 (47\%) estuvieron activos en el transcurso de las cuatro semanas de duración del curso. En estos se encontraron distintos niveles de participación que se describen a continuación:

1. Terminar $(3,471)$ : participantes fuertemente comprometidos para dominar los materiales, trabajando a través de ejercicios y obteniendo un certificado.

2. Realizar $(1,598)$ : participantes fuertemente comprometidos para dominar el material del curso observando videos principalmente. 
3. Explorar $(1,647)$ : participantes que navegaron por distintos espacios del curso para revisar los materiales.

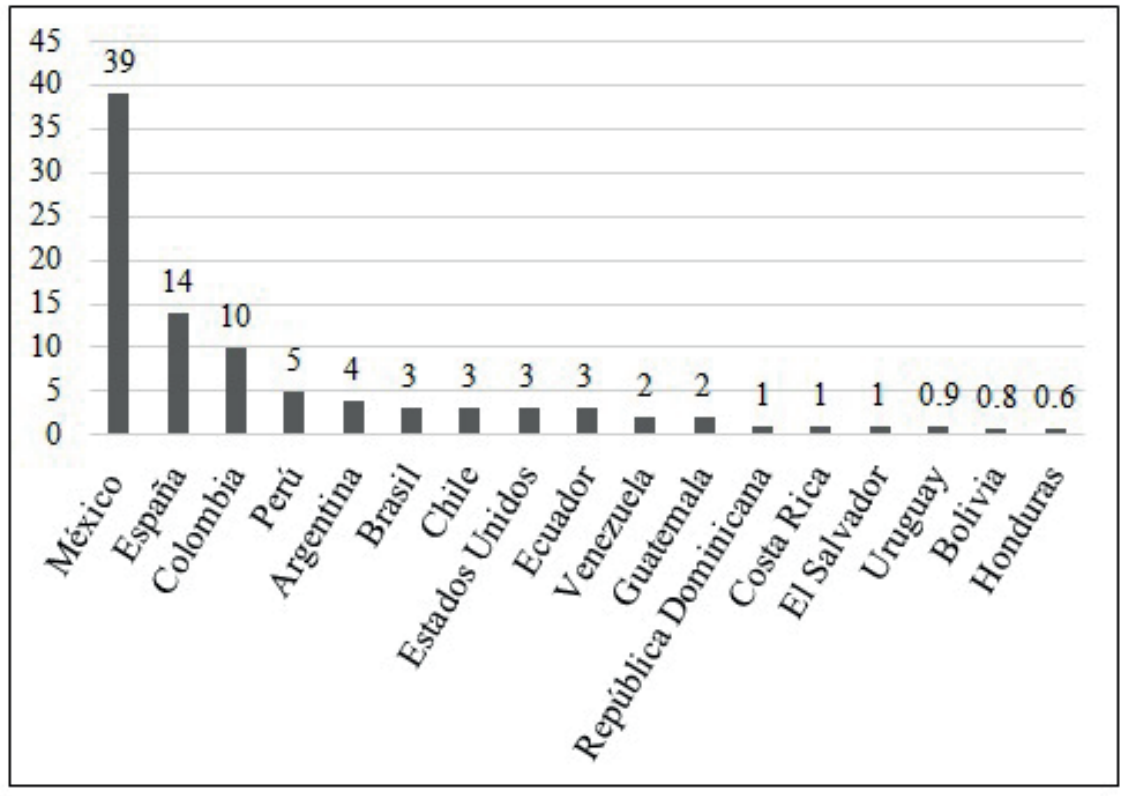

Figura 1. Difusión del curso

Fuente: Elaboración propia.

La participación en el curso tuvo un efecto decreciente conforme el paso de las cuatro semanas de duración. La primera semana registró la mayor cantidad de participantes, con 5,111 realizando algún tipo de actividad, la segunda con 2,195, la tercera con 1,171 y la cuarta con 853. De acuerdo con estos estadísticos, la mayor participación se registró alrededor del tema Movimiento Educativo Abierto, que incluía las temáticas de la innovación educativa, el acceso abierto, los REA y las tendencias y estrategias en el Movimiento Educativo Abiertos ligadas a las necesidades sociales, por lo que se considera el tema con mayor difusión. Además, la difusión se realizó predominantemente a través de los videos de expertos (Figura 2), los cuales tenían por objetivo reflexionar desde la perspectiva de tres expertos internacionales (Canadá, Chile y Portugal) sobre la validez académica de los contenidos en acceso abierto. Los videos de expertos 
contaron con los siguientes estadísticos: 57,602 vistas totales, 11,864 descargas totales y 4,735 participantes que hicieron uso del recurso.

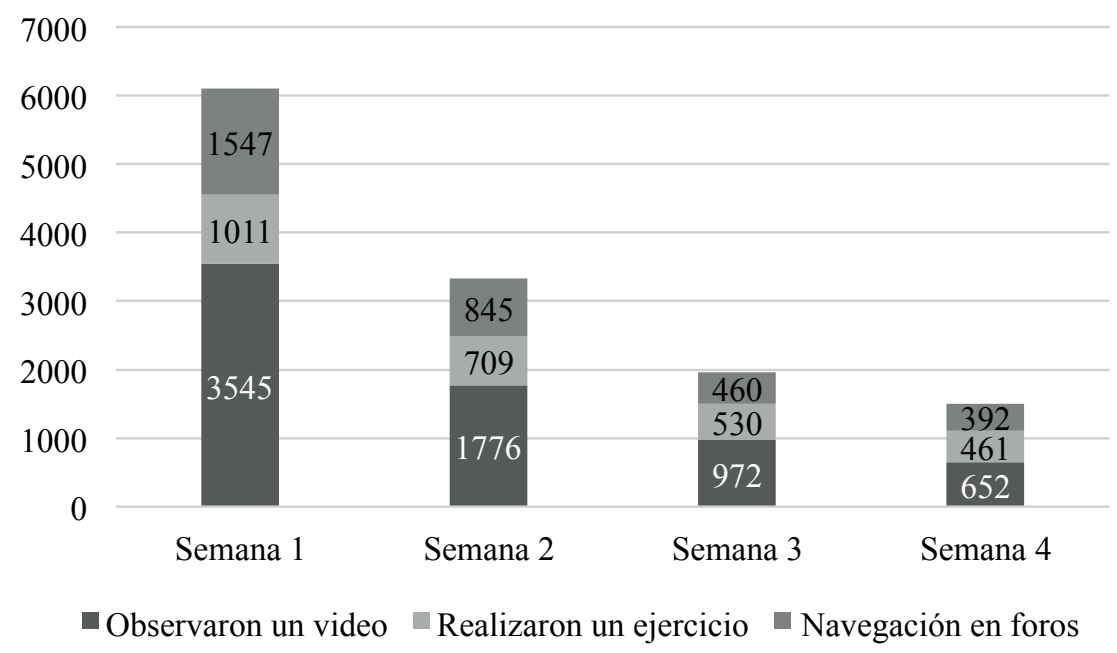

Figura 2. Tipo de actividad por semana

Fuente: Elaboración propia.

\subsection{Aprendizaje}

Al inicio del curso, se preguntó a los participantes sobre los aprendizajes que esperaban obtener de su intervención en éste. Del corpus codificado, resultó que el código otras competencias fue el de mayor predominancia en las respuestas de los participantes (Tabla 2). Esto implica que los participantes percibieron en el curso una oportunidad para desplegar ciertas competencias asociadas al uso de la tecnología, tales como la comunicación en línea, el manejo de redes sociales, estrategias didácticas para cursos en línea, uso de las plataformas virtuales de aprendizaje y el desarrollo de investigación en el tema de innovación educativa. Sobre las competencias asociadas a la utilización de REA, la movilización (33\%) fue la competencia que los participantes señalaron como su principal expectativa de aprendizaje, seguido de la producción (14\%). De tal manera, los participantes esperan, al finalizar el curso, poder producir y hacer uso de REA en su práctica profesional. 
Tabla 2. Expectativas de aprendizaje

\begin{tabular}{lc}
\hline \multicolumn{1}{c}{ Códigos } & $\begin{array}{c}\text { Número de extractos } \\
\text { de texto codificados }\end{array}$ \\
\hline Producción & $42(14.0 \%)$ \\
\hline Búsqueda y selección & $38(12.6 \%)$ \\
\hline Difusión & $9(3.0 \%)$ \\
\hline Movilización & $99(33.0 \%)$ \\
\hline Otras competencias & $110(36.6 \%)$ \\
\hline Texto no codificado & $2(0.6 \%)$ \\
\hline (respuestas descontextualizadas)
\end{tabular}

* Se calcularon sobre 300 respuestas a la encuesta de la semana 1. Fuente: Elaboración propia.

De la segunda semana en adelante, se preguntó a los participantes acerca de los aprendizajes más importantes que obtuvieron en cada una de las semanas (Tabla 3 ). Con relación a ello, se encontró una correspondencia entre las expectativas y la satisfacción del aprendizaje. Sin embargo, esta correspondencia tuvo lugar principalmente en las competencias búsqueda y selección y en la difusión de REA, que aunque se mencionaron en menor proporción como expectativas de aprendizaje, de acuerdo con los participantes fueron las que ampliaron en mayor medida. De la misma forma, se halló una vinculación con el desarrollo de competencias en el uso de la tecnología.

Tabla 3. Satisfacción sobre el aprendizaje

\begin{tabular}{lc}
\hline \multicolumn{1}{c}{ Códigos } & $\begin{array}{c}\text { Número de extractos } \\
\text { de texto codificados }\end{array}$ \\
\hline Producción & $25(2.7 \%)$ \\
\hline Búsqueda y selección & $237(26.3 \%)$ \\
\hline Difusión & $254(28.2 \%)$ \\
\hline Movilización & $110(12.2 \%)$ \\
\hline Otras competencias & $148(16.4 \%)$ \\
\hline Texto no codificado & $126(14.0 \%)$ \\
\hline
\end{tabular}

${ }^{*}$ Se calcularon sobre 900 respuestas a las encuestas de las semanas 2, 3 y 4. Fuente: Elaboración propia. 
Como estaba planeado en la propuesta de aprendizaje del curso, se realizó la evaluación de portafolios al final de cada semana. De esta evaluación se obtuvieron los niveles alcanzados en cada competencia planteada como objetivo del curso (Tabla 4). Los participantes alcanzaron en su mayoría niveles sobresalientes en cada una de las competencias o conocimientos. Destacan respecto al diseño de estrategias de difusión de la producción científica y académica para potenciar la transferencia de la innovación educativa con REA ( $59 \%$ en nivel sobresaliente), competencia que se vincula con la selección de espacios virtuales (redes sociales/plataformas/repositorios), considerando la población a la que se dirige el recurso y los objetivos del mismo.

\section{Tabla 4. Evaluación del aprendizaje}

\begin{tabular}{|c|c|c|c|c|}
\hline \multirow{2}{*}{$\begin{array}{c}\text { Tipos de } \\
\text { portafolio }\end{array}$} & \multirow{2}{*}{ Competencias } & \multicolumn{3}{|c|}{ Nivel de logro } \\
\hline & & Insuficiente & Aceptable & Sobresaliente \\
\hline \multirow{3}{*}{$\begin{array}{l}\text { Portafolio } \\
\text { diagnóstico } \\
\text { ( } 667 \text { evaluados) }\end{array}$} & $\begin{array}{l}\text { Conceptualización del Movimiento } \\
\text { Educativo Abierto }\end{array}$ & $19 \%$ & $38 \%$ & $43 \%$ \\
\hline & $\begin{array}{l}\text { Identificación de una innovación } \\
\text { educativa en el ambiente de trabajo }\end{array}$ & $21 \%$ & $36 \%$ & $44 \%$ \\
\hline & Evidencia de portafolio diagnóstico & $22 \%$ & $34 \%$ & $44 \%$ \\
\hline \multirow{3}{*}{$\begin{array}{l}\text { Portafolio de } \\
\text { trabajo } \\
\text { ( } 520 \text { evaluados) }\end{array}$} & $\begin{array}{l}\text { Acceder y usar con ética y legalidad la } \\
\text { información }\end{array}$ & $18 \%$ & $31 \%$ & $50 \%$ \\
\hline & Búsqueda de REA & $13 \%$ & $34 \%$ & $54 \%$ \\
\hline & Evidencia de portafolio de trabajo & $13 \%$ & $33 \%$ & $54 \%$ \\
\hline \multirow{2}{*}{$\begin{array}{l}\text { Portafolio de } \\
\text { evaluación } \\
\text { (445 evaluados) }\end{array}$} & Diseño de estrategias para la difusión & $9 \%$ & $30 \%$ & $59 \%$ \\
\hline & Evidencia del portafolio de evaluación & $11 \%$ & $30 \%$ & $59 \%$ \\
\hline \multirow{4}{*}{$\begin{array}{l}\text { Portafolio de } \\
\text { presentación } \\
\text { (451 evaluados) }\end{array}$} & Datos de identificación de la audiencia & $5 \%$ & $18 \%$ & $78 \%$ \\
\hline & Datos de identificación del área de impacto & $8 \%$ & $29 \%$ & $63 \%$ \\
\hline & Plantea objetivos & $10 \%$ & $26 \%$ & $64 \%$ \\
\hline & Acciones & $8 \%$ & $32 \%$ & $60 \%$ \\
\hline
\end{tabular}

Fuente: Elaboración propia.

También son capaces de buscar, seleccionar y evaluar ( $54 \%$ en el nivel sobresaliente) todo tipo de recursos en la red (lecturas/podcast/videos/software/objetos de apren- 
dizaje/cursos). Analizando sus características, determinan si se trata de un REA, así como sus posibilidades de uso sin incurrir en problemas de derechos de autor, tomando en cuenta criterios como el uso ético y legal y de validación de la información.

En cuanto a la movilización de REA, competencia que se asocia con la utilización y reutilización de recursos de un contexto a otro, son capaces de diseñar actividades para integrarlos en su contexto particular, considerando la audiencia $(78 \%$ en nivel sobresaliente) y área de impacto ( $63 \%$ en nivel sobresaliente), planteando objetivos (64\% en nivel sobresaliente) y acciones ( $60 \%$ en nivel sobresaliente) específicos. Dado que el portafolio de presentación es la máxima evidencia de aprendizaje, el análisis de los resultados sugiere que los participantes pueden hacer un uso sistemático de REA al interior de su práctica profesional. De la evaluación, se obtuvo que los portafolios sí reflejan los aprendizajes establecidos por el curso para tal actividad, por lo que representan evidencias del conocimiento obtenido por el participante durante su paso por el curso.

\section{Conclusiones}

Aunque el análisis e interpretación de los resultados se hacen sobre un caso, tienen el objetivo de fomentar la reflexión acerca de la pertinencia de un моoс como un espacio para la enseñanza y difusión de los REA, esperando aportar evidencias que informen sobre el tema en cuestión.

Sobre la difusión de los REA, se encontró que el curso tuvo un alcance casi global en el continente americano, principalmente en países como México (39\%) y Colombia (10\%). A nivel europeo, España (14\%) fue el país donde el curso alcanzó una mayor difusión. Esta conclusión se da con base en los 14,227 participantes registrados en el curso, los cuales tienen origen en 101 países distintos. Sin embargo, es importante destacar que la cantidad de participantes registrados no asegura completamente la difusión. Al respecto, la participación activa es un indicador importante para asegurarla, ya que hace referencia a los participantes que sí están expuestos a los contenidos del curso. En este sentido, la difusión del curso está relacionada con la misma enseñanza, de la cual los participantes se benefician al realizar algún tipo de actividad. Dado que el objetivo del curso es fomentar y desarrollar habilidades en el uso de REA, el análisis de la difusión y la enseñanza no se puede disociar. Desde este punto de vista, se considera significativa la difusión que tuvo el curso, ya que fueron 6,716 participantes los 
que estuvieron activos realizando actividades en relación con los REA, alcances que no se pueden lograr mediante otros tipos de formación, tales como la presencial o incluso la formación en línea en su formato tradicional.

Sobre el aprendizaje, se identificó una concatenación entre las expectativas de aprendizaje de los participantes y lo que realmente aprendieron al finalizar el curso. Las cuatro competencias básicas del Movimiento Educativo Abierto se desplegaron dentro del espacio del curso, lo que implica no sólo el conocimiento sobre dónde encontrar y cómo valorar REA, sino también, sobre cómo usarlos. Es importante señalar que la movilización de REA presentó niveles sobresalientes en todos los portafolios de aprendizaje, en particular, en el portafolio de presentación, el cual es un plan diseñado por el participante para utilizar REA de forma sistemática en su contexto profesional. De tal manera, el curso fue efectivo en promover el uso de REA; esto tiene relevancia ya que el uso o la práctica profesional con REA, es uno de los retos que aún enfrenta el Movimiento Educativo Abierto.

Finalmente, aunque las PEA engloban distintas prácticas que van más allá de la utilización de REA, el conocimiento y la utilización de este tipo de recursos son el principal camino hacia las PEA, por lo que se considera que el curso aportó en este sentido. Por otro lado, la difusión de los REA tiene ciertas ventajas cuando se da por un proceso de enseñanza. Al estar los contenidos/conocimientos asociados a una propuesta de aprendizaje, la cual es mediada por distintas actividades y espacios de socialización, da la pauta no sólo para enseñar teoría, sino también, para la práctica. Además, la difusión se incrementa cuando se utiliza un моoc.

\section{Lista de referencias}

Atkins, D., Brown, J., \& Hammond, A. (Febrero, 2007). A review of the Open Educational Resources (OER) Movement: achievements, challenges, and new opportunities (Reporte). Recuperado de https://www.hewlett.org/wp-content/ uploads/2016/08/ReviewoftheOERMovement.pdf

Deimann, M., \& Farrow, R. (2013). Rethinking OeR and their use: Open education as Bildung. The International Review of Research in Open and Distributed Learning, 14(3), 344-360. doi:10.19173/irrodl.v14i3.1370

Ehlers, U. (2011). From Open Educational Resources to Open Educational Practices. eLearning Papers, 23, 1-8. Recuperado de https://www.openeducationeuropa. 
eu/en/article/From-Open-Educational-Resources-to-Open-EducationalPractices?paper $=72110$

Guárdia, L., Maina, M., \& Sangrá, A. (2013). Mooc Design Principles. A Pedagogical Approach from the Learner's Perspective. eLearning Papers, 33. Recuperado de https://www.openeducationeuropa.eu/en/article/MOOC-Design-Principles.A-Pedagogical-Approach-from-the-Learner\% $\mathrm{E}_{2} \% 80 \%$ g9s-Perspective

Lakhan, S., \& Jhunjhunwala, K. (2008). Open Source Software in Education. Educause Quarterly, 31(2), 32-40. Recuperado de https://er.educause.edu/ /media/files/article-downloads/eqmo824.pdf

Liyanagunawardena, T., Adams, A., \& Williams, S. (2013). Moocs: a Systematic Study of the Published Literature 2008-2012. International Review of Research in Open and Distributed Learning, 14(3), 202-227. Recuperado de http://www. irrodl.org/index.php/irrodl/article/view/1455/2531

McAndrew, P. (2011). Fostering Open Educational Practices. eLearning Papers, 23. Recuperado de https://www.openeducationeuropa.eu/en/article/FosteringOpen-Educational-Practices?paper $=72110$

McAndrew, P., \& Farrow, R. (2013). Open Education Research: From the Practical to the Theoretical. En R. McGreal, W. Kinuthia \& S. Marshall (Coords.), Open Educational Resources: Innovation, Research and Practice (pp. 65-78). Canadá: Commonwealth of Learning and Athabasca University. Recuperado de http://oasis.col.org/bitstream/handle/11599/486/pub_PS_OER-IRP web. pdf? sequence $=1$ \&is Allowed $=y$

Organización de las Naciones Unidas para la Educación, la Ciencia y la Cultura. (2002). Forum on the Impact of Open Courseware for Higher Education in Developing Countries (Reporte CI-2002/CONF.803/CLD.1). Recuperado de http://unesdoc.unesco.org/images/o012/001285/128515e.pdf

Ramírez, M. S. (Coord.). (2013). Competencias Docentes y Prácticas Educativas Abiertas en Educación a Distancia. México: Lulu.

Ramírez, M. S. (2015). Acceso abierto y su repercusión en la Sociedad del Conocimiento: Reflexiones de casos prácticos en Latinoamérica. Education in the Knowledge Society, 16(1), 103-118. doi:10.14201/eks2015161103118

Stake, R. (1999). Investigación con estudio de casos. Madrid: Morata.

Wiley, D. (2010). Openness as Catalyst an Educational Reformation. Educause Review Online, 45(4). Recuperado de https://er.educause.edu/articles/2010/8/ openness-as-catalyst-for-an-educational-reformation 


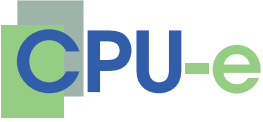

Enseñanza y difusión sobre el uso de recursos

educativos abiertos con MOOC: un estudio de caso

Martín Alonso Mercado Varela, Katiuska Fernández Morales, Gilles Lavigne, María Soledad Ramírez Montoya

Yiotis, K. (2005). The open access initiative: A new paradigm for scholarly communications. Information, Technology and Libraries, 24(4), 157-162. doi:10.6017/ital. v24i4.3378 